\title{
Hypoxia-challenged MSC-derived exosomes deliver miR-210 to attenuate post-infarction cardiac apoptosis
}

\section{CURRENT STATUS: UNDER REVIEW}

Stem Cell Research \& Therapy $\triangle B M C$

Hao Cheng

Zhongshan Hospital Fudan University

- jxchenghao@139.comCorresponding Author

ORCiD: https://orcid.org/0000-0003-1360-6290

Shufu Chang

Zhongshan Hospital Fudan University

Rende Xu

Zhongshan Hospital Fudan University

Lu Chen

Zhongshan Hospital Fudan University

Xiaoyue Song

Zhongshan Hospital Fudan University

Jian Wu

Zhongshan Hospital Fudan University

Juying Qian

Zhongshan Hospital Fudan University

Yunzeng Zou

Zhongshan Hospital Fudan University

Jianying $\mathrm{Ma}$

Zhongshan Hospital Fudan University

DOI:

10.21203/rs.3.rs-18180/v1

\section{SUBJECT AREAS}

Stem Cell \& Developmental Cell Biology 
KEYWORDS

Myocardial infarction; exosome; mesenchymal stem cells; hypoxia; apoptosis 
Abstract

Background

Myocardial infarction (MI) is a major cause of death worldwide. Although percutaneous coronary intervention and coronary artery bypass grafting can prolong life, cardiac damage persists. In particular, cardiomyocytes have no regenerative capacity. Mesenchymal stem cells (MSCs) are attractive candidates for the treatment of MI. The manner by which MSCs exert a beneficial effect upon injured cells is a source of continued study.

Methods

After the isolation and identification of exosomes from MSCs, the expression of miR-210 was determined by Microarray Chip. Subsequently, gain- and loss-function approaches were conducted to detect the role of exosomes and exosomal-miR-210 in cell proliferation and apoptosis of cardiomyocytes, as well as the MI in vivo. Dual-Luciferase Report Gene System was used to demonstrate the target gene of miR-210.

Results

We tested the hypothesis that MSC-derived exosomes transfer specific miRNA to protect cardiomyocytes from apoptotic cell death. Interestingly, direct cardiac injection of MSC exosomes reduced infarct size and improved heart function after coronary ligation. In vitro, the MSC exosomes enhanced cardiomyocyte survival to hypoxia. Confirmation of exosome uptake in myocytes was confirmed. Dual luciferase reporter assay implicated miR-210 as a mediator of the therapeutic effect and AIFM3 as a downstream target. Treatment with miR-210 overexpressing MSC exosomes improved myocyte protection to both in vitro and in vivo stress. Furthermore, the endogenous and exogenous miR-210 had the same therapeutic effects.

\section{Conclusion}

These results demonstrated that the beneficial effects offered by MSC-exosomes transplantation after $\mathrm{MI}$ are at least partially because of excreted exosome containing mainly miR-210.

Full Text

Due to technical limitations, full-text HTML conversion of this manuscript could not be completed. 
However, the manuscript can be downloaded and accessed as a PDF.

Figures 
A
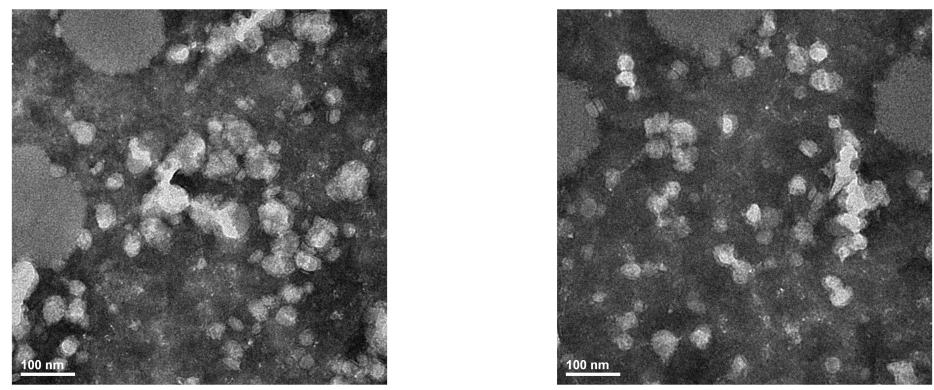

B
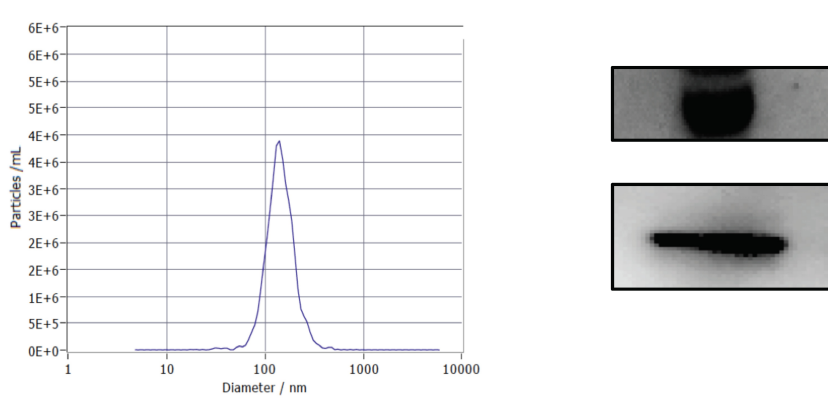

CD63 ( $26 \mathrm{kDa}$ )

TSG101 ( 44 kDa)

C ( 
Figure 1

Characterization of exosomes derived from MSCs. A. Transmission electron micrographs of exosomes. Scale bar, $100 \mathrm{~nm}$. Arrows, representative individual exosomes. B. Nanoparticle Tracking Analysis(NTA) of exosomes. C. Western blot analysis of CD63 and TSG101 expression in exosomes. Each experiment was repeated 3 times. 

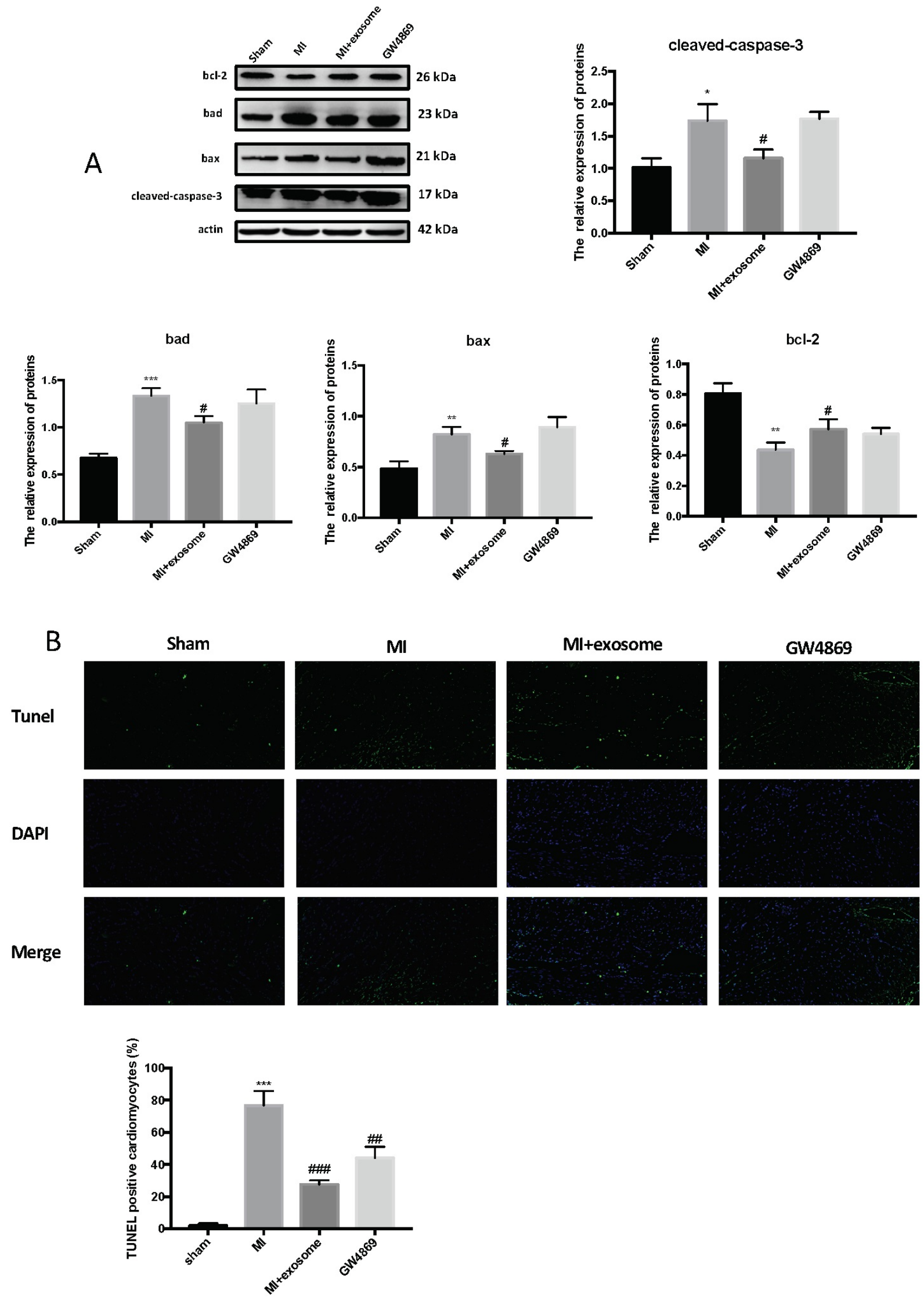
Figure 2

Exosomes derived from MSCs inhibit apoptosis after myocardial infarction. A. Expression levels of the apoptotic proteins BAD, BAX, BCL-2, and Cleaved-CASPASE-3 were evaluated in cardiac tissue sections from sham-operated rats and coronary-ligated rats that received either no therapy (PBS), MSC exosomes, exosomes from MSCs treated after vessel occlusion with GW4869 immediately ( $\mathrm{N}=5$ per group). Western blot analysis of protein lysates obtained from the infarct border zone. Quantitative analysis of BAD, BAX, BCL-2, and Cleaved-CASPASE-3 is shown in the lower panel. B. Cell injury was determined by TUNEL assay. Fluorescence staining with vital dyes shown in blue indicates live cardiomyocytes, whereas ethidium homodimer-1 staining labels dead cells green. The percentage of cardiomyocytes death is shown in the right panels. Each experiment was repeated 3 times. $* \mathrm{p}<0.05, * * \mathrm{p}<0.01, * * * \mathrm{p}<0.001$ for MI vs. sham. $\# \mathrm{p}<0.05$ for $\mathrm{MI+exosome} \mathrm{vs.} \mathrm{MI.}$ 

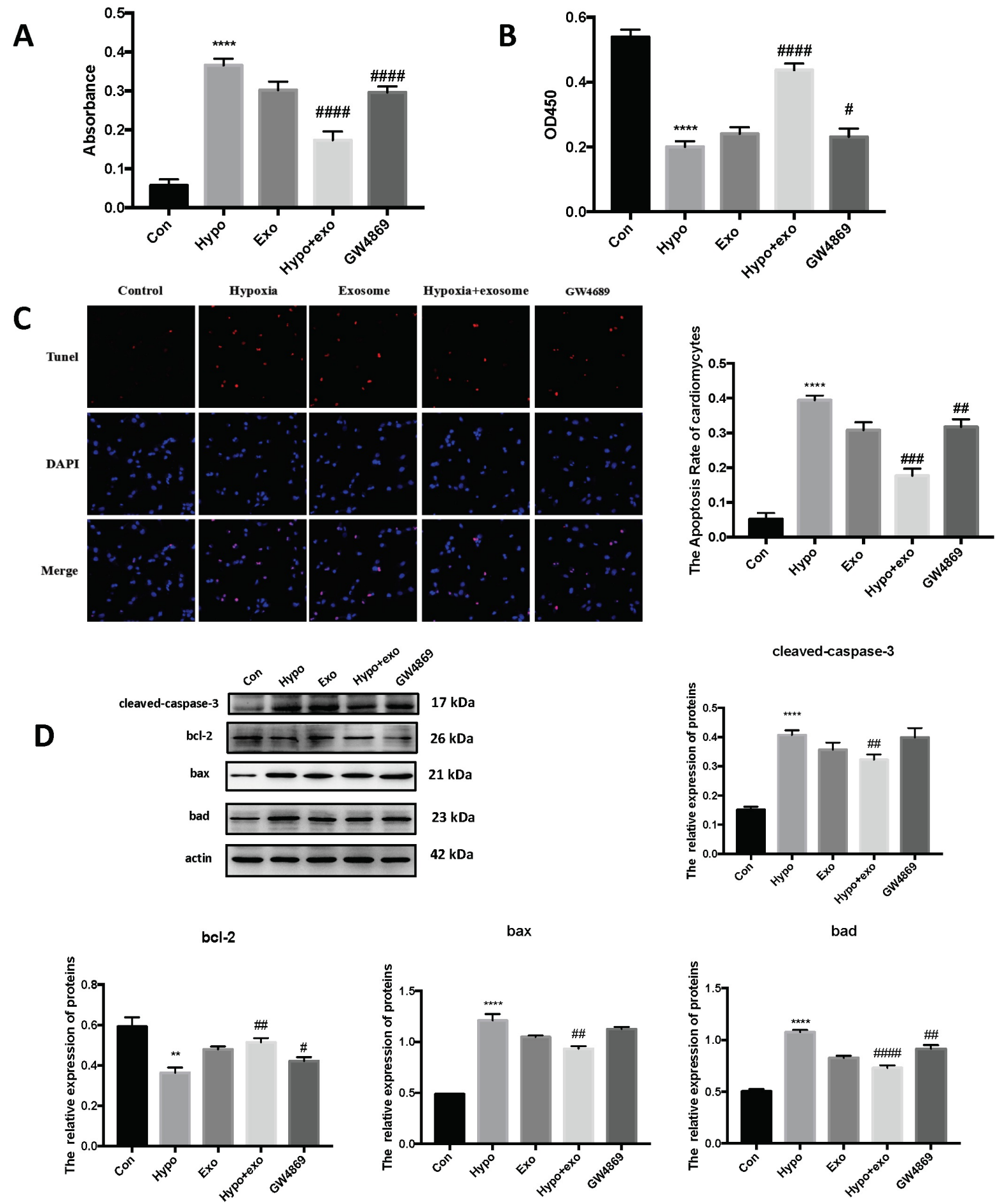
Figure 3

Exosomes derived from MSCs reduce cell death. Hypoxic cardiomyocytes cultured in $1 \% 02$ for 24 hours and treated with the indicate agents. Cell injury was determined by LDH release assay (A), MTT assay (B) and TUNEL assay (C). Fluorescence staining with vital dyes shown in green indicates live cardiomyocytes, whereas ethidium homodimer-1 staining labels dead cells red. The percentage of cardiomyocytes death is shown in the right panels. D. BAD, BAX, BCL-2 and Cleaved-CASPASE-3 levels were evaluated by Western blot and expression quantified. Each experiment was repeated 3 times. ${ }^{*} p<0.01, * * * * p<0.0001$ for Hypo vs. Con. \#\#\#\#p<0.0001 for Hypo+exo or GW4869 vs. Hypo. 


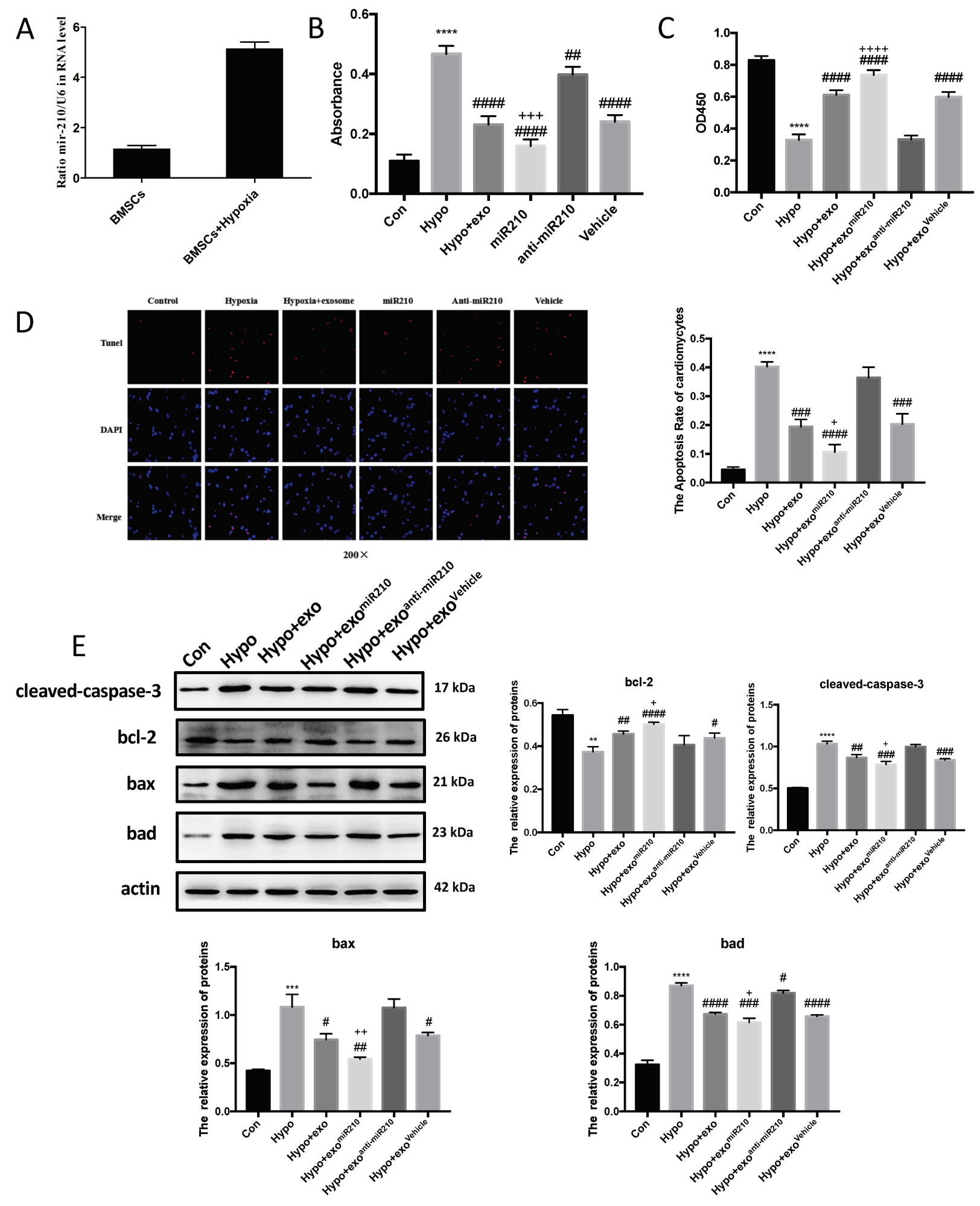


Figure 4

Overexpression of miR210 in MSC exosomes strengthens their cardioprotective function in vitro. A. Polymerase chain reaction quantification of miR210 in the exosomes obtained from MSCs. Hypoxic cardiomyocytes were treated with exosomes obtained from MSCs that were pre-treated with miR210 (exomiR210), anti-miR210 (exoanti-miR210) or scramble (exoVehicle) for the indicted time and cell injury characterized by LDH release (B), viability by MTT assay (C) and apoptotic programmed cell death by TUNEL assay (D). Red, TUNELpositive nuclei; blue, DAPI-stained nuclei; green, Troponin-positive cardiomyocytes. Scar bar $200 \times$. The percentage of TUNEL-positive cells is shown in the right panels E. Western blot identification of BAD, BAX, BCL-2, and Cleaved-CASPASE-3 in cardiomyocyte lysates after cell incubation with the indicated exosomes \pm hypoxia. Quantitative analysis of BAD, BAX, BCL-2 and Cleaved-CASPASE-3 is shown in the lower panel. Each experiment was repeated 3 times. ${ }^{*} p<0.01, * * * p<0.001, * * * * p<0.0001$ for Hypo vs. Con. $\# p<0.05, \# \# p<0.01$, \#\#\#p<0.001, \#\#\#\#p<0.0001 for Hypo+exo, Hypo+exomiR210, Hypo+exoanti-miR210 or Hypo+exoVehicle vs. Hypo. $+p<0.05,++p<0.01,+++p<0.001++++p<0.0001$ for Hypo+exomiR210 vs. Hypo+exo. 

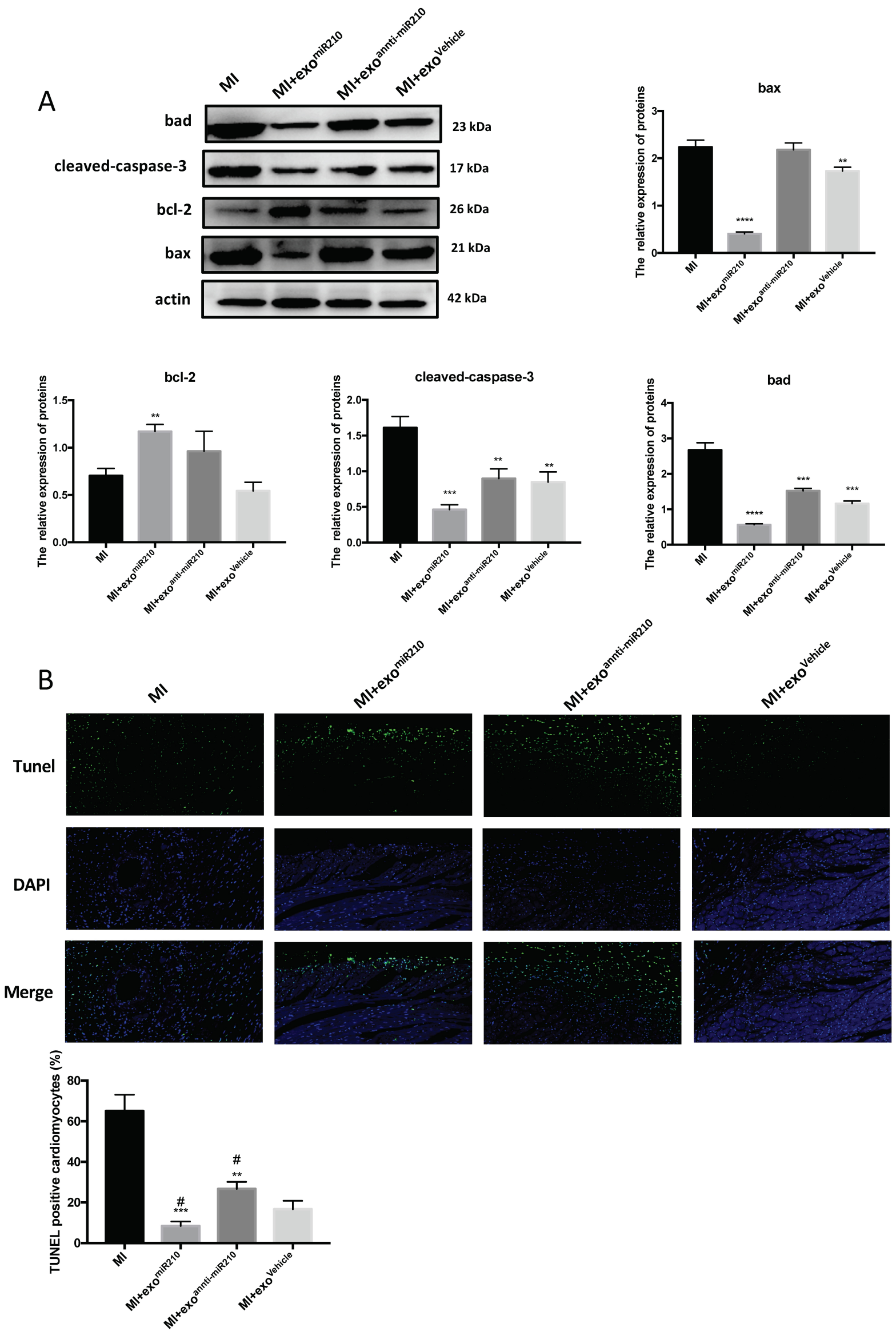
Figure 5

Exosomes derived from MSCs with miR-210 overexpression inhibit apoptosis after myocardial infarction. A. Western blot analysis of protein levels of apoptosis-associated genes BAD, BAX, BCL-2, and Cleaved-CASPASE-3 from cardiac tissue samples from mice in the indicated treatment groups. Each experiment was repeated 3 times. B. Cell death in tissue samples was characterized by TUNEL assay. ${ }^{* *} p<0.01, * * * p<0.001, * * * * p<0.0001$ for MI+exomiR210, MI+exoanti-miR210, MI+exoVehicle vs. MI. \#p<0.05 for MI+exomiR210, MI+exoanti-miR210 vs. MI+exoVehicle. 
A

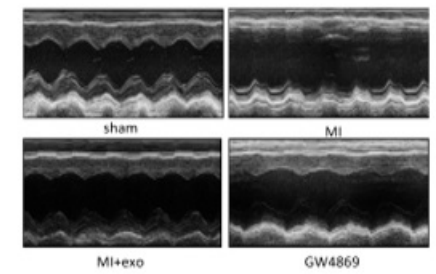

$B$
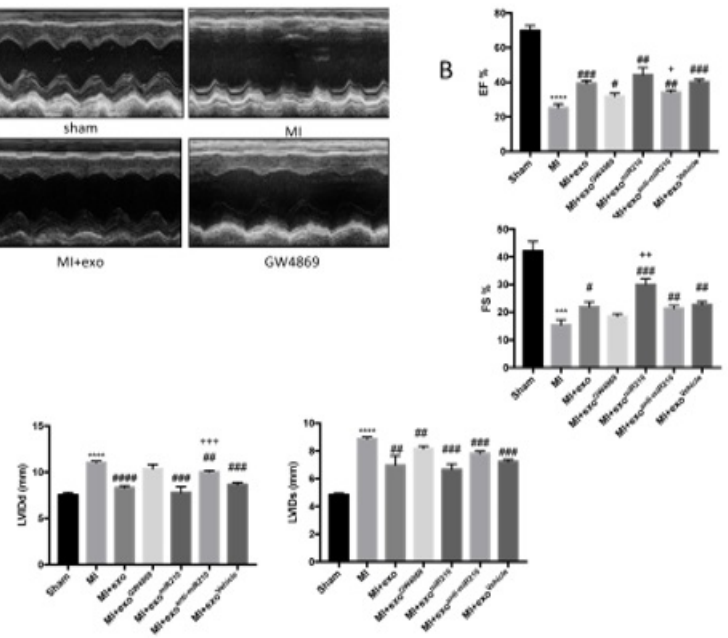

GW4869

D

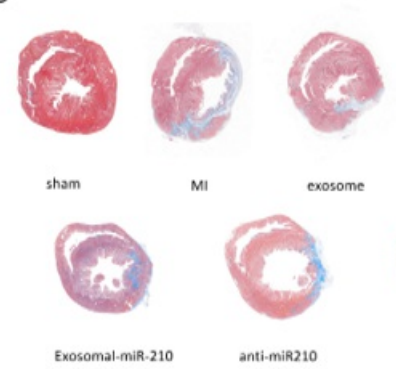

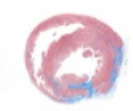

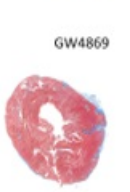

vehicle

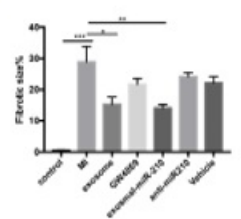

m

$E$

sham
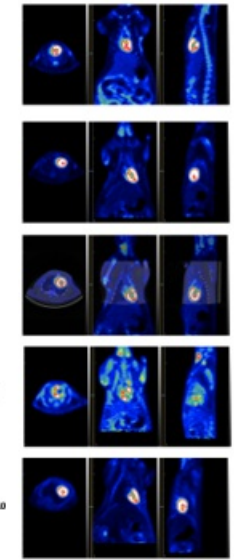

Mirexomentis

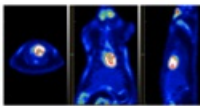

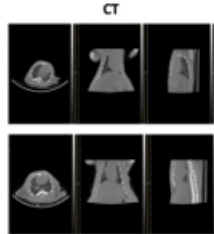

(6) 1
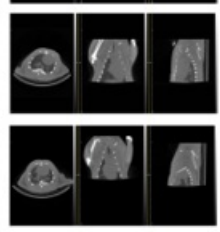

(a) 4.4

ol 2
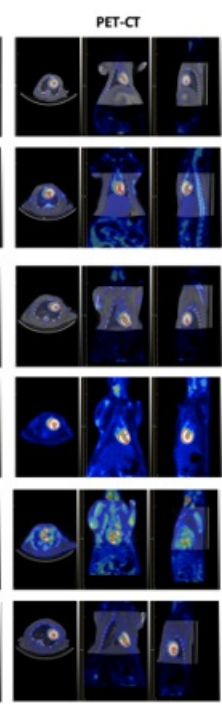

0 (6)

Figure 6

Overexpression of miR-210 in MSC exosomes leads to improvement of cardiac function and infarct size in vivo at 1 month after MI. A-C. Representative photographs of M-mode echocardiography. Quantitative analysis of echocardiography ( $N=4$ per group). FS, fractional shortening; EF, ejection fraction; LVIDd, left ventricular internal diameter enddiastolic; LVIDs, left ventricular internal diameter end-systolic. D. Masson Trichrome staining of whole heart tissue sections 28 days post-ligation and treatment with PBS, MSC exosomes, exosomes derived from MSCs treated with GW4869, MSCs-exomiR210, MSCs-exoantimiR210, or MSCs-exoVehicle. Percentage of tissue fibrosis is quantified and presented. $\mathrm{N}=$ 4 per group. E. Representative photographs of PET-CT of rats from the respective treatment groups. $\mathrm{N}=3$ per group. ${ }^{* * *} \mathrm{p}<0.001, * * * * \mathrm{p}<0.0001$ for $\mathrm{MI}$ vs. sham. \# $\mathrm{p}<0.05, \# \# \mathrm{p}<$ 0.01, \#\#\# $p<0.001$, \#\#\# $p<0.0001$ for MI+exosome, GW4869, MSCs-exomiR210, MSCs-exoanti-miR210, MSCs-exoVehicle vs. MI. $+p<0.05,++p<0.01,+++p<0.001$ for MSCs-exomiR210, MSCs-exoanti-miR210 vs. MSCs-exoVehicle. 


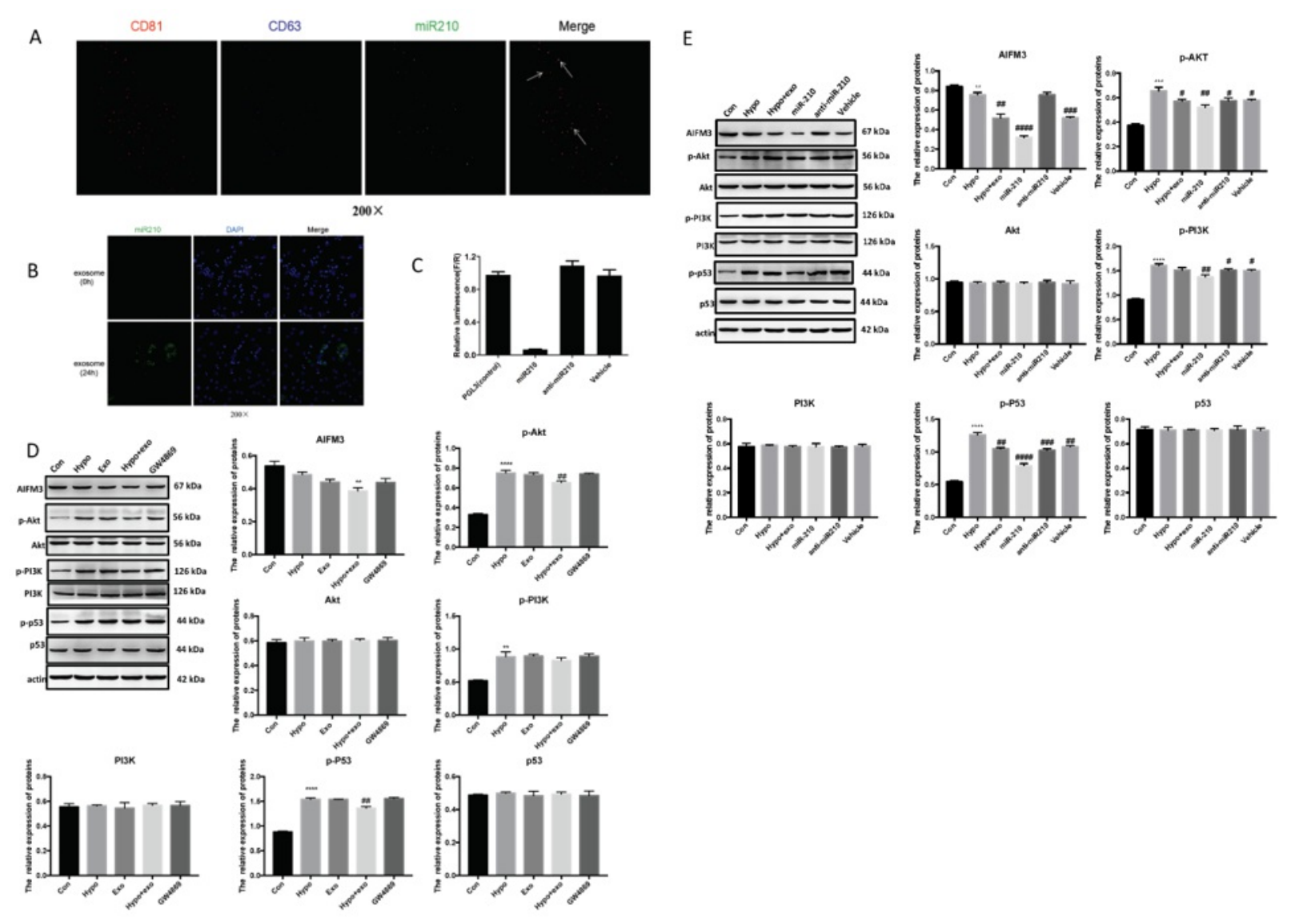

Figure 7

Hypoxia-mediated apoptosis is associated with changes in PI3K and AKT. A. Co-localization of miR-210 with exosome-specific surface marker CD81 and CD63 by the immunofluorescence. B. The uptake of exosome by cardiomyocytes, and the localization of miR-210 molecule with Cy3 fluorescence in cardiomyocytes. C. dual luciferase reporter assay showed that AIFM3 was a target of miR-210. F, Firefly luciferase; R, Renilla luciferase. D\&E. AIFM3, p-AKT/AKT, p-PI3K/PI3K, p-p53/p53 levels were evaluated by Western blotting in cardiomyocytes from the indicated treatment groups \pm hypoxia. Quantifications are shown in the lower panel. $\mathrm{N}=3$ independent experiments. ${ }^{* *} \mathrm{p}<0.01, * * * * p<0.0001$ for Hypo vs. con. \#\#p<0.01 for Hypo+exo vs. Hypo. 

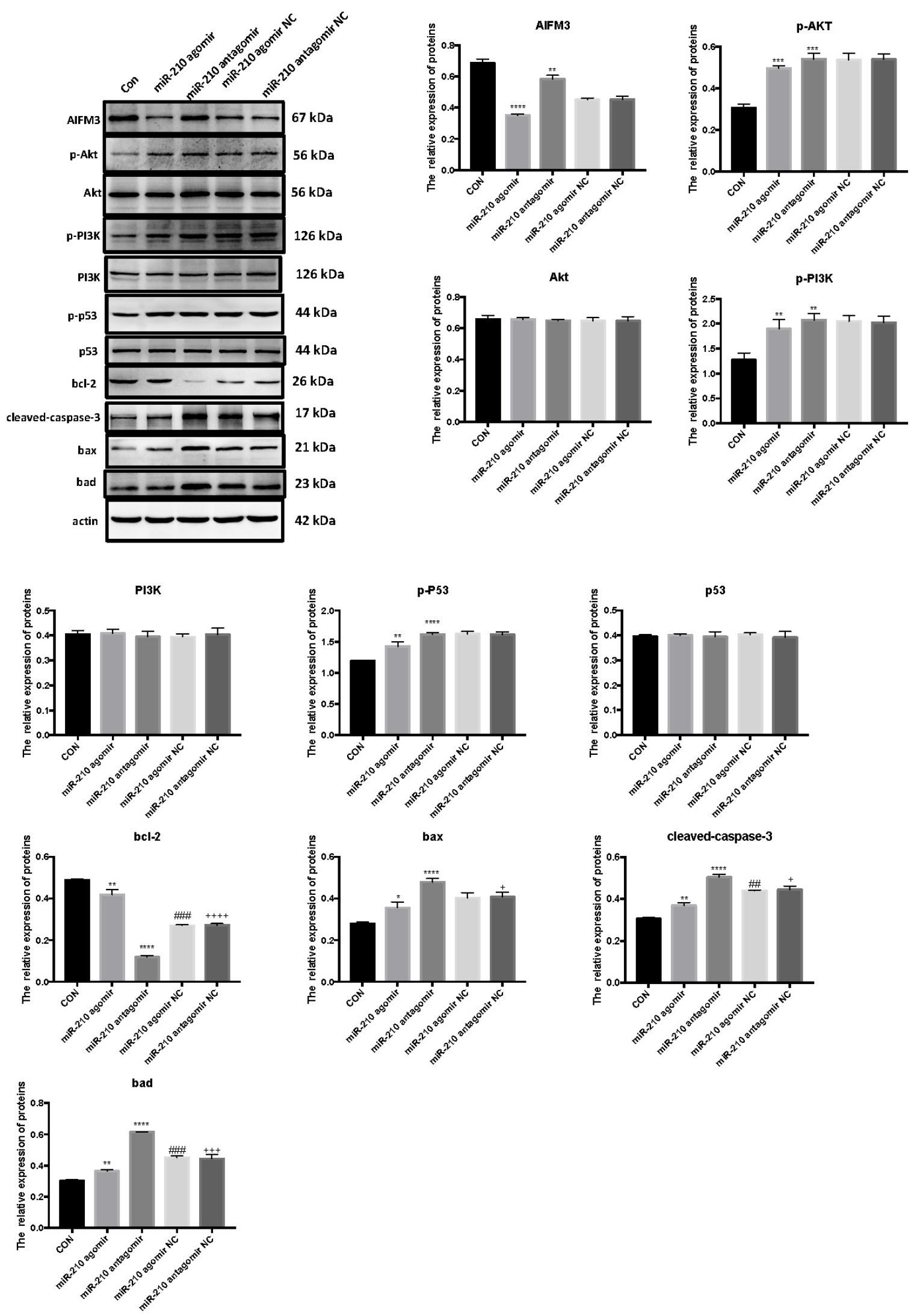
Figure 8

Comparison of the uptake of exosomal miR-210 with the regulatory activity of endogenous miR-210. The protein levels of AIFM3, p-AKT/AKT, p-PI3K/PI3K, p-p53/p53, BAD, BAX, BCL-2, and Cleaved-CASPASE-3, were evaluated by Western blotting in cardiomyocytes treated with overexpressed miR-210. Quantifications are shown in the lower panel. $N=3$ independent experiments. $* * \mathrm{p}<0.01, * * * \mathrm{p}<0.001, * * * * \mathrm{p}<0.0001$ for miR-210 agomir and miR-210 antagomir vs. con. \#\# $p<0.01$, \#\#\# $p<0.001$ for miR-210 agomir NC vs. miR-210 agomir. $+p<0.05,+++p<0.001,++++p<0.0001$ for miR-210 antagomir NC vs. miR-210 antagomir.

\section{Supplementary Files}

This is a list of supplementary files associated with this preprint. Click to download. Supplemental Information.pdf 